\title{
Sequential Effects of Increasing Propofol Sedation on Frontal and Temporal Cortices as Indexed by Auditory Event-related Potentials
}

Wolfgang Heinke, M.D., ${ }^{*}$ Ramona Kenntner, M.Sc., † Thomas C. Gunter, Ph.D., $\ddagger$ Daniela Sammler, M.Sc.,§

Derk Olthoff, M.D., Ph.D., Prof., || Stefan Koelsch, Ph.D.\#

\begin{abstract}
Background: It is an open question whether cognitive processes of auditory perception that are mediated by functionally different cortices exhibit the same sensitivity to sedation. The auditory event-related potentials $P 1$, mismatch negativity (MMN), and early right anterior negativity (ERAN) originate from different cortical areas and reflect different stages of auditory processing. The $\mathbf{P 1}$ originates mainly from the primary auditory cortex. The MMN is generated in or in the close vicinity of the primary auditory cortex but is also dependent on frontal sources. The ERAN mainly originates from frontal generators. The purpose of the study was to investigate the effects of increasing propofol sedation on different stages of auditory processing as reflected in P1, MMN, and ERAN.

Methods: The P1, the MMN, and the ERAN were recorded preoperatively in 18 patients during four levels of anesthesia adjusted with target-controlled infusion: awake state (target concentration of propofol $0.0 \mu \mathrm{g} / \mathrm{ml})$, light sedation $(0.5 \mu \mathrm{g} / \mathrm{ml})$, deep sedation $(1.5 \mu \mathrm{g} / \mathrm{ml})$, and unconsciousness $(2.5-3.0 \mu \mathrm{g} / \mathrm{ml})$. Simultaneously, propofol anesthesia was assessed using the Bispectral Index.

Results: Propofol sedation resulted in a progressive decrease in amplitudes and an increase of latencies with a similar pattern for MMN and ERAN. MMN and ERAN were elicited during sedation but were abolished during unconsciousness. In contrast, the amplitude of the P1 was unchanged by sedation but markedly decreased during unconsciousness.

Conclusion: The results indicate differential effects of propofol sedation on cognitive functions that involve mainly the auditory cortices and cognitive functions that involve the frontal cortices.
\end{abstract}

RECENT findings indicate that cognitive processes mediated by functionally different cortices (e.g., association cortex $v s$. primary sensory cortex) are differentially affected by anesthetic drugs. ${ }^{1-4}$ Nevertheless, investigations related to these phenomena are sparse, and it is an

\footnotetext{
* Research Associate, || Head, Department of Anesthesiology and Intensive Care Therapy, University of Leipzig. † Research Associate, Max Planck Institute of Cognitive Neuroscience, and Department of Psychology, University of Würzburg. $\ddagger$ Research Associate, Max Planck Institute of Cognitive Neuroscience. Research Associate, Max Planck Institute of Cognitive Neuroscience, and Department of Psychology, University of Leipzig. \# Junior Research Director, Max Planck Institute of Cognitive Neuroscience, and Harvard Medical School.

Received from the Departments of Anesthesiology and Intensive Care Therapy and Psychology, University of Leipzig, Leipzig, Germany; the Max Planck Institute of Cognitive Neuroscience, Leipzig, Germany; the Department of Psychology, University of Würzburg, Würzburg, Germany; and Harvard Medical School, Boston, Massachusetts. Submitted for publication April 18, 2003. Accepted for publication September 22, 2003. Supported by Verein zur Förderung der Forschung und Fortbildung in der Anästhesiologie, Intensiv-, Schmerz- und Notfalltherapie e.V., Leipzig, Germany. The work of Drs. Kenntner, Sammler, and Koelsch is attributed to the Max Planck Institute of Cognitive Neuroscience, Leipzig, Germany.

Address reprint requests to Dr. Heinke: Klinik und Poliklinik für Anästhesiologie, und Intensivtherapie, Universität Leipzig, Liebigstraße 20a, 04103 Leipzig, Germany. Address electronic mail to: heiw@medizin.uni-leipzig.de. Individual article reprints may be purchased through the Journal Web site, www.anesthesiology.org.
}

open question whether anesthesia has different effects on primary auditory processes and auditory processes that involve other than auditory cortices. Therefore, the current study investigates auditory event-related brain potentials (AERPs) during systematic sedation. The recorded AERPs reflect well-defined processing stages of the acoustic input that are mediated by different cortical structures.

The P1, the frequency mismatch negativity (MMN), and the early right anterior negativity (ERAN) are AERPs that can be recorded even in the absence of attention, ${ }^{5-8}$ which is a prerequisite to assess effects of anesthesia. The P1 reflects sensory encoding of auditory stimulus attributes. ${ }^{5,9}$ The frequency MMN mainly reflects auditory sensory memory operations. It is elicited by deviant auditory stimuli in a series of standard stimuli. ${ }^{5,6}$ The ERAN can be elicited by music syntactic violations, that is, violations of regularities of a culture's musical repertoire. ${ }^{10}$ Therefore, ERAN and MMN reflect higher cognitive operations than the neural processes reflected in the P1.

The P1 is generated within or in the close vicinity of the primary auditory cortex ${ }^{11}$ (located in the temporal lobe), whereas the MMN receives its main contributions also from the primary and periprimary auditory cortical regions but is also dependent on activity of the frontal cortices. ${ }^{12-15}$ The ERAN has been reported to be generated mainly in the frontal cortical areas. ${ }^{16}$ Therefore, similar effects of gradually progressing propofol sedation on the three AERPs indicate that the frontal and auditory cortices are affected similarly by propofol, whereas different effects on the three AERPs indicate that the frontal and auditory cortices are affected differently.

The Bispectral Index (BIS) is increasingly used to monitor depth of anesthesia. Investigations of relations between the BIS and the changes in late AERPs (latencies > $80 \mathrm{~ms}$ ) with increasing propofol sedation have not been reported. They may reveal the BIS values at which distinct cognitive processes (e.g., auditory memory, sound identification, language comprehension) collapse. Such information may help to avoid intraoperative awareness. Previous studies related to this phenomenon indicate that even accurate language processing was unaffected in the majority of patients who reported intraoperative auditory perceptions. ${ }^{17}$ With this respect, the approach of investigating music syntactic processing during sedation seems intriguing because language and music are assumed to be processed in overlapping cerebral networks. ${ }^{18-20}$ Therefore, music experiments may also help 
to reveal at which anesthetic depth language functions collapse.

The current study investigates P1, MMN, and ERAN during progressive propofol sedation while simultaneously recording the BIS. Stronger effects of propofol on the ERAN than on the MMN were expected because the ERAN mainly originates from frontal cortices that mediate the complex processing of (musical) structure, whereas the MMN originates mainly from the auditory cortex (although along with contributions from the frontal cortical structures). In contrast to the MMN and the ERAN, we expected the P1 to be less affected by propofol sedation because the P1 originates from primary auditory cortical regions and reflects more basic auditory processes.

\section{Materials and Methods}

\section{Subjects}

Eighteen patients (age, 18 - $48 \mathrm{yr}$; mean age, $30.4 \mathrm{yr}$; six female) with American Society of Anesthesiologists physical status I or II who were scheduled to undergo elective arthroscopic surgery participated in the study. Exclusion criteria included a history of deafness, obesity, hiatus hernia, or psychiatric disorder. All subjects were nonmusicians (they had never learned a musical instrument and had no special musical education besides typical school education). The study was approved by the local ethics committee of the Medical Faculty, University of Leipzig (Leipzig, Germany). Written informed consent was obtained from each subject on the day before the measurement.

\section{Experimental Design and Procedure}

Anesthesia. The experiment took place directly before the planned operation, outside the operating room in a comfortable environment. The amount of background noise was kept to a minimum, and the lights were dimmed. No sedative or other premedication was applied. On arrival in the preanesthetic care unit, the patients were routinely prepared for anesthesia. An intravenous catheter was placed into a forearm vein for drug administration. The electrocardiogram and arterial oxygen saturation were continuously monitored during the entire experiment, and noninvasive blood pressure was recorded every $5 \mathrm{~min}$. Participants breathed air throughout the study. If necessary, upper airway obstruction was relieved by gentle skin support. Participants were instructed to relax and to keep their eyes closed during the entire experimental session.

Auditory event-related potentials were recorded during four different levels of anesthesia. The order of these levels was fixed. By means of target-controlled infusion of propofol (Disoprifusor ${ }^{\circledR}$; Becton Dickinson Infusion Systems, Brezins, France), the following blood plasma

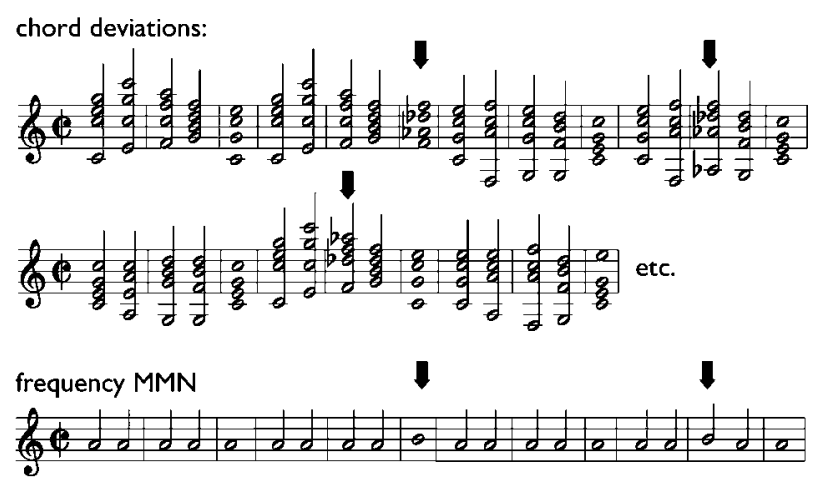

Fig. 1. Example of stimuli: music block (suited to elicit an early right anterior negativity, top and center rows) and tone block (suited to elicit a mismatch negativity, bottom row). In all blocks, stimuli were presented with the same time course, loudness, and probability of deviant events (deviant events are indicated by arrows).

levels were targeted: level A (awake, $0 \mu \mathrm{g} / \mathrm{ml}$ ), level B (light sedation, $0.5 \mu \mathrm{g} / \mathrm{ml}$ ), level C (deep sedation, $1.5 \mu \mathrm{g} / \mathrm{ml}$ ), and level D (unconsciousness, 2.5-3.0 $\mu \mathrm{g} /$ $\mathrm{ml}$ ). The AERP recording during each level started $8 \mathrm{~min}$ after the concentration of propofol was increased. This time delay is necessary for the equilibration between the targeted plasma concentration of propofol and the effect site concentration of propofol (i.e., the concentration of propofol in the brain). ${ }^{21}$ In addition, the effect of propofol was evaluated on-line using the Bispectral Index ${ }^{\circledR}$ monitor (A-2000 BIS ${ }^{\circledR}$ monitor, system version 2.10, BIS algorithm 3.4; Aspect Medical Systems, Natick, MA). This procedure guaranteed that AERP recording was started only if sedation was sufficiently deepened at a stable anesthetic level.

BIS values were recorded for analysis at 3, 6, 9, 12, and 15 min after starting the experiment during each level. A clinical assessment of anesthesia was obtained by verbal communication and tactile stimulation at the end of each level. After the experiment was finished, the patient was moved into the operating room and prepared for surgery.

Stimuli. Two blocks were presented during each level of sedation: a block with musical stimuli (ERAN block) and an auditory oddball paradigm suited to elicit the frequency MMN (MMN block). Each level of sedation had a duration of 16:30 min. Synthesizer-generated stimuli (Roland JV2080; Roland Corporation, Hamamatsu, Japan) were presented via headphones. Each stimulus sequence during each experimental block (ERAN, MMN) consisted of five events (chords in the ERAN block and single tones in the MMN block; fig. 1). Stimulus sequences were presented in direct succession; there were no silent periods between events or sequences. All chords or tones had the same decay of loudness. ${ }^{7}$

ERAN Block. Stimuli were identical to those used in previous studies (see Koelsch et al. ${ }^{7}$ Maess et al. ${ }^{16}$ and Koelsch et $a l^{22}$ for detailed description). One hundred 
sixty chord sequences, each sequence comprising five chords, were presented during each level of sedation. The presentation time of chords 1 to 4 of each sequence was $600 \mathrm{~ms}$, and the fifth chord was presented for 1,200 ms. Music syntactically regular chords served as standard stimuli, whereas music syntactically irregular chords (Neapolitan sixth chords, presented at either the third or the fifth position of a chord sequence) served as deviant stimuli (fig. 1). Sequences were selected in a way that $25 \%$ of the sequences contained a Neapolitan chord at the third position and $25 \%$ contained a Neapolitan chord at the fifth position, resulting in 40 Neapolitans at the third and 40 Neapolitans at the fifth position in each level (block duration was approximately $11 \mathrm{~min}$ ). Note that Neapolitan chords violate the musical structure, or musical syntax, and that Neapolitan chords at the fifth position represent a stronger music syntactic violation than at the third position: Neapolitans at the third position are relatively suitable with respect to their chord function (Neapolitans may be interpreted as a subdominant variation and usually precede a dominant seventh chord), and Neapolitans presented at the end of a chord sequence instead of a tonic strongly violate music structural regularities. The degree of music syntactic violation is reflected in the amplitude of the ERAN (in listeners familiar with the major-minor tonal system): Neapolitans at the fifth position evoke an ERAN with a larger amplitude than Neapolitans at the third position. ${ }^{7,10,22}$

MMN Block. Stimuli of the MMN block were presented with the same time course, loudness, and arrangement of deviant tones as the stimuli of the ERAN block: Sequences of five tones were used; the standard tone had a frequency of $440 \mathrm{~Hz}$ (fig. 1). Ten percent of all tones were frequency deviants $(496 \mathrm{~Hz})$; deviants were presented at the third position $(P=0.25)$ and the fifth position $(P=0.25)$, resulting in 40 deviant tones presented at each level. Note that it has been shown previously that, in contrast with the ERAN, the amplitude of the MMN elicited at the fifth position does not differ from the amplitude of the MMN elicited at the third position. ${ }^{10}$ Therefore, deviants from the third and fifth position could be collapsed, resulting in a block duration of only approximately 5:30 min

AERP Recording and Data Analysis. Electroencephalographic data were measured with $\mathrm{Ag}-\mathrm{AgCl}$ electrodes (digitized with a sampling rate of $250 \mathrm{~Hz}$ ) from the scalp using the following 18 electrode positions of the international 10-20 system: A1 (auricular left) and A2 (auricular right), F3 and F7 (frontal left), F4 and F8 (frontal right), FZ (frontocentral), FT7 (frontotemporal left), FT8 (frontotemporal right), FC3 (frontocentral left), FC4 (frontocentral right), C3 (central left), C4 (central right), $\mathrm{CZ}$ (centrocentral), T7 (temporal left), T8 (temporal right), $\mathrm{CP} 5$ (centroparietal left), and CP6 (centroparietal right; fig. 2). Because participants were lying in the supine position during the experiment, no elec-
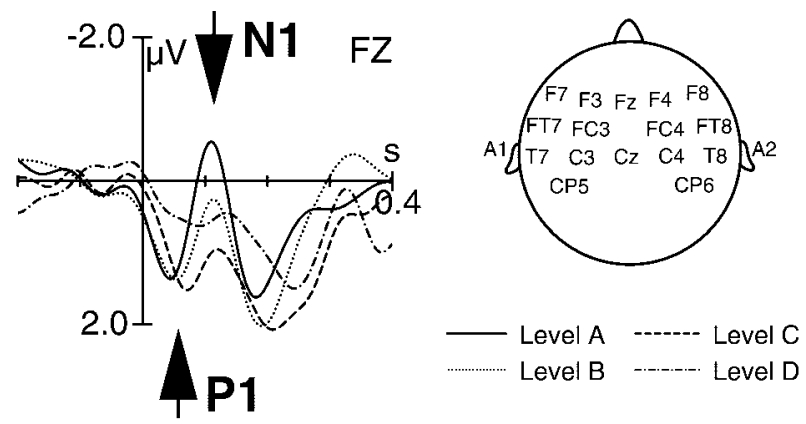

Fig. 2. Grand average waveforms of $P 1$ and $N 1$ elicited at FZ during awake state (level A), light sedation (level B), deep sedation (level C) and unconsciousness (level D). Note that the P1 potential (and the N1 potential) was computed by averaging standard stimuli only. The vertical line indicates the stimulus onset. The amplitude of the P1 was unaffected by sedation but markedly reduced during propofol-induced unconsciousness. The image of the bead depicts the electrode positions used for recording of the electroencephalographic data.

trodes were placed over parietal and occipital areas. The electrode sites measured are sufficient for a valid identification of P1, MMN, and ERAN ${ }^{6-8}$ and no source localization by means of current source density analysis was intended. An electrode placed on the nose tip served as a reference, and the ground electrode was located on the chest. To control for artifacts caused by eye movements, vertical and horizontal electrooculograms were recorded bipolarly.

After the measurement, electroencephalograms were band-pass filtered $(0.5-10 \mathrm{~Hz}, 1,001$ points, finite impulse response). For artifact reduction, electroencephalographic data were rejected off-line from the raw electroencephalogram whenever the SD of the electroencephalographic signal recorded any electrode exceeded $50 \mu \mathrm{V}$ within a 200 -ms or 600 -ms gliding window (this procedure was applied for each electrode). Rejections were visually inspected and corrected (if necessary) by the first two authors.

Epochs (from -200 to $600 \mathrm{~ms}$, with respect to stimulus onset) were averaged off-line from the artifact-reduced electroencephalographic data using a 200-ms prestimulus baseline. Then, the single-subject AERPs were grand averaged across all subjects for each condition. Grand-averaged AERPs were inspected visually for the presence of ERAN and MMN. On the basis of the literature, ${ }^{5-7}$ differences between standard and deviant stimuli were only regarded as MMN or ERAN if they fulfilled the following criteria: (1) at frontal electrodes, the AERP of deviant stimuli had to be more negative than that of the standard stimuli; (2) at central electrodes, effects of deviants (compared with effects of standards) had to be less negative than at frontal electrodes (that is, both ERAN and MMN had to be smaller at the central electrode $\mathrm{CZ}$ than at the frontal electrode FZ); (3) at mastoid electrodes (electrode positions A1 and A2), effects had to invert polarity; and (4) maximum amplitude of effects 
Table 1. Physiologic Parameters

\begin{tabular}{|c|c|c|c|c|c|c|}
\hline Level of Sedation & $\mathrm{HR}$, beats/min & Systolic BP, mmHg & Diastolic BP, mmHg & Mean $\mathrm{BP}, \mathrm{mmHg}$ & $\mathrm{SaO}_{2}, \%$ & BIS \\
\hline A: awake state & $72 \pm 12$ & $148 \pm 13$ & $84 \pm 14$ & $105 \pm 12$ & $97 \pm 1$ & $95.4 \pm 2.95$ \\
\hline B: light sedation & $71 \pm 14$ & $143 \pm 14$ & $82 \pm 13$ & $101 \pm 15$ & $97 \pm 1$ & $87.6 \pm 7.45^{\star}$ \\
\hline C: deep sedation & $71 \pm 15$ & $136 \pm 13^{*}$ & $78 \pm 9$ & $96 \pm 10$ & $96 \pm 2^{*}$ & $72.1 \pm 7.11^{\star}$ \\
\hline D: unconsciousness & $72 \pm 14$ & $128 \pm 14^{*}$ & $74 \pm 11$ & $93 \pm 10^{*}$ & $95 \pm 1^{*}$ & $50.4 \pm 6.74^{*}$ \\
\hline
\end{tabular}

Physiologic data (mean $\pm \mathrm{SD} ; \mathrm{n}=18$ ).

* Significant differences from baseline values (level $A$, awake state; $P<0.05$ ).

$\mathrm{BIS}=$ Bispectral Index; $\mathrm{BP}=$ blood pressure; $\mathrm{HR}=$ heart rate; $\mathrm{SaO}_{2}=$ arterial oxygen saturation.

had to be between 90 and $250 \mathrm{~ms}$. Moreover, negative effects of Neapolitan chords at the fifth position in the ERAN block were only regarded as ERAN when effects were larger at the fifth than at the third position (proving that effects were not only elicited by the physically deviant out-of-key notes of Neapolitans).

Visual observations were tested statistically by computing the mean amplitudes at frontal electrodes (F3, FZ, F4) in time windows determined on the basis of visual inspection (time windows were centered around the maxima of effects) and previous studies ${ }^{6,10,22}$ : level A: P1 30-70 ms, MMN 100-160 ms, ERAN 140-200 ms, P3a 250-300 ms; level B: P1 35-75 ms, MMN 110-170 ms, ERAN 150-210 ms, P3a 250-300 ms;

level C: P1 50-90 ms, MMN 120-180 ms, ERAN 160220 ms; and level D: P1 55-95 ms, MMN 130-190 ms, ERAN 170-230 ms.

Neither MMN nor ERAN was observed at level D; however, for statistical purposes, a time window had to be chosen, and taking into account the prolongation of the latency of the AERPs during levels A-C, the time windows used for level $\mathrm{D}$ are the most reasonable ones. AERPs were statistically analyzed by repeated-measures analysis of variance (ANOVA) with four factors: level of sedation (A-D), stimulus type (ERAN, MMN), position within sequence (third $v s$. fifth), and deviance (deviant, standard).

\section{Results}

\section{Physiologic Parameters and Depth of Anesthesia}

The changes in the physiologic variables are shown in table 1. ANOVAs were conducted to reveal differences in heart rate, blood pressure, arterial oxygen saturation, and BIS values. The group average evaluation of the physiologic variables shows significant decreases in arterial oxygen saturation, systolic blood pressure, and mean blood pressure at level C (deep sedation) and level D (unconsciousness) compared with the awake state caused by propofol infusion $(P<0.05)$. Heart rate and diastolic blood pressure did not change significantly with increasing sedation.

The BIS value decreased significantly from level to level $(P<0.05)$. The average BIS value during wakeful- ness (level A) was 95.4 (SD, 2.95). During light propofol sedation (level B), most patients were slightly tired and more relaxed compared with during wakefulness. Normal verbal communication was always possible; the speech of the patients was unaffected. Two (of 18) patients fell into light sleep but were easily woken up at the end of the level. Four patients reported feeling unaffected by the drug. The mean of the recorded BIS values of level B (light sedation) was 87.6 (SD, 7.45). During deep sedation (level C), most of the patients seemed to be asleep. At the end of this level, 10 patients were difficult to wake up by verbal commands (i.e., they responded only after their name was called repeatedly with a loud voice), whereas 8 patients were immediately responsive. The speech of the patients was delayed and slurred. The mean BIS value of all patients was 72.1 (SD, 7.11). The immediately responsive patients at the end of level C had a mean BIS value of 76.5 (SD, 6.99), whereas the patients with delayed response were significantly more sedated $(P<0.05)$ as reflected by a lower mean BIS value of 69.3 (SD, 6.45). During drug-induced unconsciousness (level D), none of the patients showed any response to loud verbal commands or tactile stimulation. The mean BIS value of level D was 50.4 (SD, 6.74).

\section{Event-related Potentials: P1}

The P1s elicited by in-key chords and standard tones (from all positions of the stimulus sequences) are shown in figure 2. A clear P1 was observed at all levels of sedation. The P1 amplitudes were virtually identical during the awake state and sedation (levels A-C) but markedly reduced during unconsciousness (level D) (fig. 2 and tables 2 and 3). An ANOVA with factor sedation (A-D) revealed an effect of propofol on the amplitude of the P1 $\left(\mathrm{F}_{3.51}=6.98, P<0.05\right)$. Further ANOVAs comparing the $\mathrm{P} 1$ amplitudes elicited during levels $\mathrm{A}$ and $\mathrm{B}$, $A$ and $C$, and $B$ and $C$ revealed no difference between the respective levels $(P>0.75$ in each test). In contrast, ANOVAs comparing the P1s elicited during levels $\mathrm{A}$ and $\mathrm{D}, \mathrm{B}$ and $\mathrm{D}$, and $\mathrm{C}$ and $\mathrm{D}$ revealed significant effects $(P<$ 0.008 in each test) indicating that the P1 elicited in an unconscious state (level D) differs from the P1 elicited during wakefulness and sedation (levels A-C). 
Table 2. Summary of AERP ANOVAs

\begin{tabular}{|c|c|c|c|}
\hline Levels & AERP Components & Factors & $P$ Value \\
\hline$A-D$ & $(\mathrm{MMN}+\mathrm{ERAN}), \mathrm{P1}$ & Component $\times$ sedation & $0.0001^{*}$ \\
\hline$A-D$ & P1 & Sedation & $0.05^{\star}$ \\
\hline$A, B$ & $\mathrm{P} 1$ & Sedation & 0.79 \\
\hline$A, C$ & $\mathrm{P} 1$ & Sedation & 0.75 \\
\hline$B, C$ & $\mathrm{P} 1$ & Sedation & 0.89 \\
\hline$A, D$ & $\mathrm{P} 1$ & Sedation & $0.007^{\star}$ \\
\hline$B, D$ & $\mathrm{P} 1$ & Sedation & $0.008^{*}$ \\
\hline$C, D$ & $\mathrm{P} 1$ & Sedation & $0.008^{*}$ \\
\hline \multirow[t]{3}{*}{$A-D$} & MMN, ERAN & Deviance & $0.0001^{*}$ \\
\hline & & Deviance $\times$ sedation & $0.0001^{*}$ \\
\hline & & Deviance $\times$ sedation $\times$ stimulus type & 0.97 \\
\hline \multirow[t]{3}{*}{$A-C$} & MMN, ERAN & Deviance & $0.0001^{*}$ \\
\hline & & Deviance $\times$ sedation & $0.0002^{*}$ \\
\hline & & Deviance $\times$ sedation $\times$ stimulus type & 0.72 \\
\hline \multirow[t]{3}{*}{$A, B$} & MMN, ERAN & Deviance & $0.0001^{*}$ \\
\hline & & Deviance $\times$ sedation & 0.14 \\
\hline & & Deviance $\times$ sedation $\times$ stimulus type & 0.45 \\
\hline \multirow[t]{3}{*}{$A, C$} & MMN, ERAN & Deviance & $0.0001^{*}$ \\
\hline & & Deviance $\times$ sedation & $0.0006^{*}$ \\
\hline & & Deviance $\times$ sedation $\times$ stimulus type & 0.62 \\
\hline \multirow[t]{3}{*}{$\mathrm{B}, \mathrm{C}$} & MMN, ERAN & Deviance & $0.0001^{*}$ \\
\hline & & Deviance $\times$ sedation & $0.005^{\star}$ \\
\hline & & Deviance $\times$ sedation $\times$ stimulus type & 0.70 \\
\hline$A-C$ & ERAN & Deviance $\times$ position & $0.0006^{*}$ \\
\hline$A-C$ & MMN & Deviance $\times$ position & 0.90 \\
\hline \multirow[t]{2}{*}{ A } & ERAN & Deviance & $0.0001^{*}$ \\
\hline & & Deviance $\times$ position & $0.03^{*}$ \\
\hline \multirow[t]{2}{*}{ B } & ERAN & Deviance & $0.0001^{*}$ \\
\hline & & Deviance $\times$ position & $0.002^{*}$ \\
\hline \multirow[t]{2}{*}{ C } & ERAN & Deviance & $0.02^{*}$ \\
\hline & & Deviance $\times$ position & $0.03^{*}$ \\
\hline A & MMN & Deviance & $0.0001^{*}$ \\
\hline$B$ & MMN & Deviance & $0.0005^{\star}$ \\
\hline C & MMN & Deviance & $0.05^{\star}$ \\
\hline \multirow[t]{2}{*}{$A, B$} & P3a & Deviance & $0.02^{*}$ \\
\hline & & Deviance $\times$ sedation & 0.95 \\
\hline
\end{tabular}

Summary of analysis of variance (ANOVAs) testing amplitude differences of auditory event-related potentials (AERPs) elicited during the four levels of sedation ( $\mathrm{A}=$ awake; $\mathrm{B}=$ light sedation; $\mathrm{C}=$ deep sedation; $\mathrm{D}=$ unconsciousness).

* Statistically significant.

ERAN = early right anterior negativity; $\mathrm{MMN}=$ mismatch negativity.

\section{Event-related Potentials: $M M N$ and ERAN}

Both MMN and ERAN were significantly elicited during wakefulness, light sedation, and deep sedation (tables 2 and 3 and figs. 3 and 4): effects of the deviant stimuli (deviant tones and irregular chords) had a maximal amplitude with negative polarity at frontal electrode sites in the time range between 100 and $230 \mathrm{~ms}$, a smaller (but still negative) amplitude over central electrodes, and a positive polarity potential ("polarity inversion") at mastoidal sites. Effects elicited by the music syntactic violations were significantly larger at the fifth than at the third position of the chord sequences (during levels A-C),

Table 3. Amplitudes and Latencies of AERPs

\begin{tabular}{|c|c|c|c|c|c|c|c|c|}
\hline \multirow[b]{2}{*}{ Level } & \multicolumn{2}{|c|}{ P1 } & \multicolumn{2}{|c|}{ MMN } & \multicolumn{2}{|c|}{ ERAN } & \multicolumn{2}{|c|}{ P3a } \\
\hline & Latency, ms & Amplitude, $\mu \mathrm{V}$ & Latency, ms & Amplitude, $\mu \mathrm{V}$ & Latency, ms & Amplitude, $\mu \mathrm{V}$ & Latency, ms & Amplitude, $\mu \mathrm{V}$ \\
\hline$A$ & 50 & $1.25 \pm 0.4$ & 130 & $2.07 \pm 1.3$ & 170 & $1.97 \pm 1.5$ & 290 & $1.44 \pm 3.6$ \\
\hline B & 55 & $1.28 \pm 0.5$ & 135 & $1.73 \pm 1.6$ & 180 & $1.82 \pm 1.1$ & 280 & $1.47 \pm 2.3$ \\
\hline $\mathrm{C}$ & 70 & $1.31 \pm 0.9$ & 135 & $0.64 \pm 1.6^{*}$ & 195 & $0.57 \pm 1.1^{*}$ & - & - \\
\hline D & 75 & $0.29 \pm 1.3^{*}$ & - & - & - & - & - & - \\
\hline
\end{tabular}

Peak latencies and mean amplitudes ( \pm SD) of $\mathrm{P} 1$, frequency mismatch negativity (MMN), early right anterior negativity (ERAN), and P3a elicited at the frontal electrodes of interest (FZ, F3, F4) in the time windows used for statistical analyses (see Materials and methods). Amplitude values of MMN, ERAN, and P3a are given as difference of the electric potentials (standard subtracted from deviant stimuli). The P3a was only observed in the MMN - block.

* Significant differences compared with level $A$ (awake state; $P<0.05$ ).

$\mathrm{AERP}=$ auditory event-related potential; $\mathrm{B}=$ light sedation; $\mathrm{C}=$ deep sedation; $\mathrm{D}=$ unconsciousness 
A

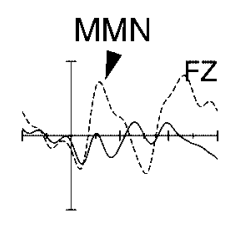

\section{MMN-Block}

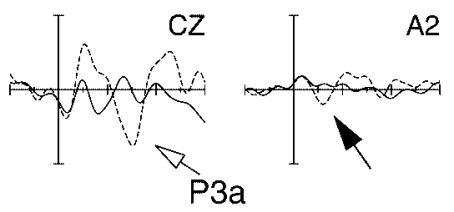

B
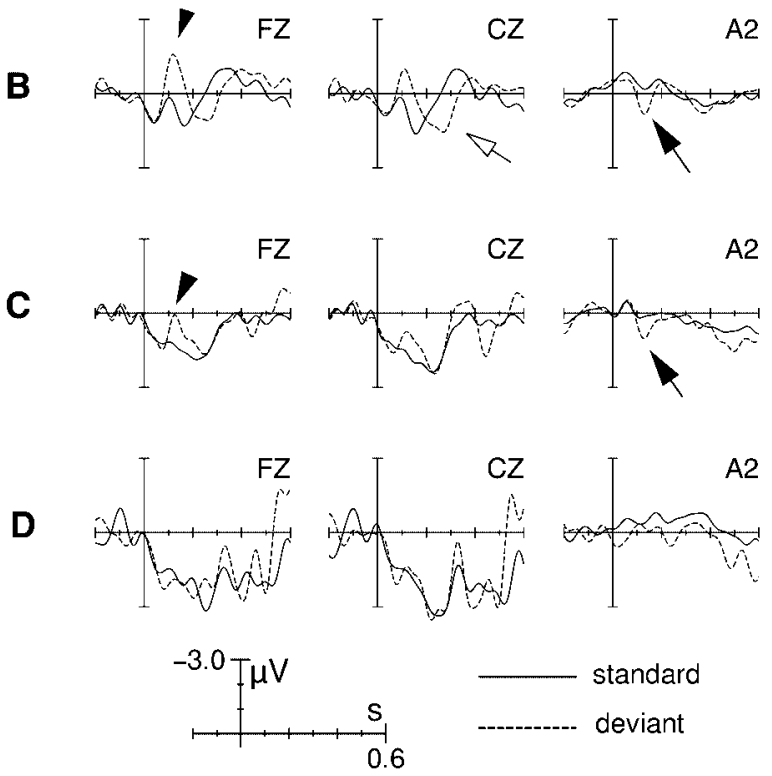

0.6

Fig. 3. Frequency mismatch negativity (MMN; grand average waveforms from $\mathrm{FZ}, \mathrm{CZ}$, and $\mathrm{A2}$ ), recorded during the awake state (level A; top row), light sedation (level B; second row), deep sedation (level $\mathrm{C}$; third row), and unconsciousness (level D; bottom row). Vertical lines indicate the stimulus onset. The short arrow indicates the MMN evoked by deviant stimuli (dotted line) compared with standard stimuli (solid line). Note the polarity inversion at mastoidal leads (indicated by the long arrow). The amplitude of the MMN was slightly decreased during light sedation, markedly decreased during deep sedation, and was abolished during unconsciousness. In levels $A$ and $B$, the MMN was followed by a P3a (indicated by the open arrows).

indicating that these effects reflect the processing of music syntactic irregularities ${ }^{10}$ (table 3; see also Materials and Methods, AERP Recording and Data Analysis, third paragraph, last sentence). No such amplitude variation between the third and fifth positions of a stimulus sequence was observed for the MMN.

From levels A to C, the amplitudes of both MMN and ERAN decreased with increasing propofol sedation (tables 2 and 3 and figs. 3 and 4); this amplitude decrease was similar for MMN and ERAN: in each level, the MMN had virtually the same amplitude as the ERAN elicited at the fifth position of the chord sequences. No ERAN was observed for harmonically irregular chords at the third position during level C (fig. 4), most presumably because of the low signal-to-noise ratio (note that the amplitude of the ERAN at the third position is expected to be smaller than at the fifth position and that the ERAN is already clearly reduced at the fifth position). Neither
MMN nor ERAN was observed during unconsciousness (level D).

As mentioned above, the patients showed differences in their anesthetic depth at the end of level C (see Results, Physiologic Parameters, and Depth of Anesthesia, second paragraph). Visual inspection yields that MMN and ERAN were clearly visible in the group average data of patients who were immediately responsive, whereas MMN and ERAN were only marginally present in the group average data of patients who showed a delayed response. However, this difference was not statistically significant. Due to the low signal-to-noise ratio of the data in level C, further experiments are needed to clarify this issue in detail.

Interestingly, during wakefulness (level A) and light sedation (level B), the MMN was followed by a significant P3a-like positive potential that was frontocentrally maximal and peaked around $280 \mathrm{~ms}$ (fig. 3). Light sedation did not affect the amplitude of the P3a $(P>0.95$; tables 2 and 3). In contrast, no P3a was observed in levels $\mathrm{C}$ or D or in any ERAN block.

To test MMN and ERAN amplitudes statistically, a global ANOVA with the factors deviance (deviant, standard), sedation (all levels, A-D), and stimulus type (tones, music) was conducted. Results indicated an effect of deviance $\left(\mathrm{F}_{1,17}=43.96, P<0.0001\right.$, reflecting that the AERPs elicited by the deviant stimuli differed from those elicited by standards), an effect of sedation $\left(\mathrm{F}_{3,51}=7.30, P<0.01\right.$, reflecting that the brain potentials were affected by sedation), and an interaction between the factors deviance and sedation $\left(\mathrm{F}_{3,51}=27.98\right.$, $P<0.0001$, reflecting that MMN and ERAN decreased in amplitude with increasing propofol sedation), but no three-way interaction $(P>0.95$, reflecting that the amplitude decrease did not differ between MMN and ERAN). To ensure that the interaction between the factors deviance and sedation was not only due to the strong decrease of MMN and ERAN in level D, an analogous ANOVA was computed only for the data of levels A-C; again, a strong interaction between the factors deviance and sedation was indicated $\left(\mathrm{F}_{2,34}=22.14, P<\right.$ $0.0002)$. To further investigate the amplitude decrease of ERAN and MMN, separate ANOVAs for the data of levels $\mathrm{A}$ and $\mathrm{B}, \mathrm{A}$ and $\mathrm{C}$, and $\mathrm{B}$ and $\mathrm{C}$ (all ANOVAs with the factors deviance, sedation, and stimulus type, as above) were conducted. A comparison of levels A versus B did not indicate an interaction between the factors deviance and sedation $(P=0.14$, reflecting that the amplitude decrease of MMN and ERAN between levels A and B was fairly small). In contrast, comparisons of levels A versus $\mathrm{C}$ and $\mathrm{B}$ versus $\mathrm{C}$ revealed clear interactions between the two factors $(P<0.005$ in each test $)$, indicating a significant amplitude decrease from level B to C. No three-way interaction was indicated in any ANOVA $(P>0.4$ in each test), reflecting that the effects of increasing propofol sedation did not differ between MMN and ERAN. 
ERAN 5th position

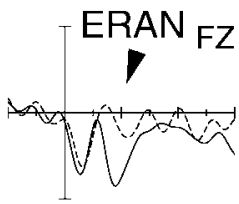

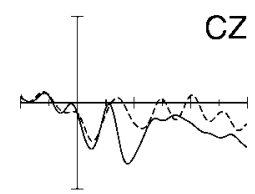

$\mathrm{CZ}$

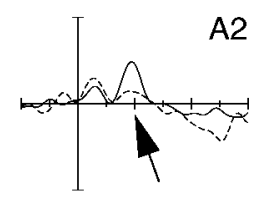

A2
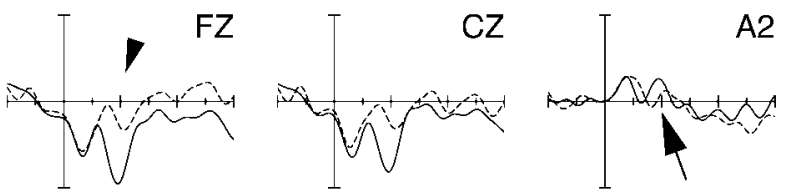

A2
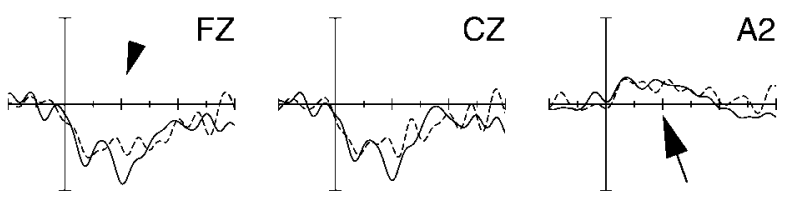

A2
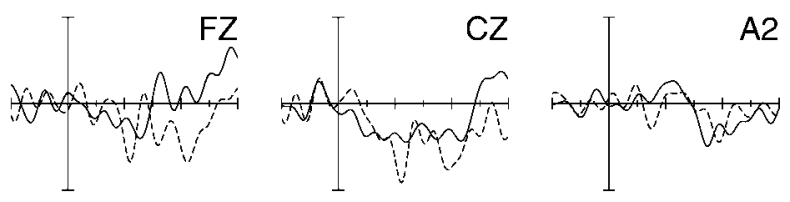

A2

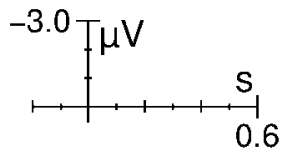

ERAN 3rd position

A

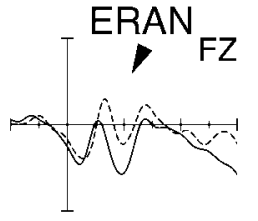

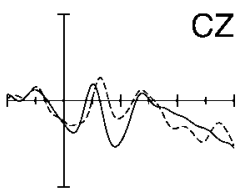

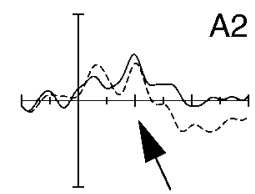

A2

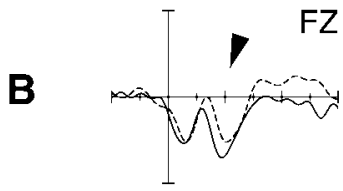

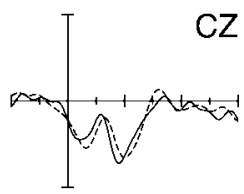

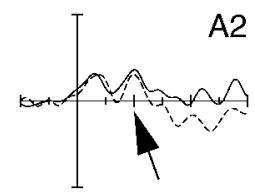

A2

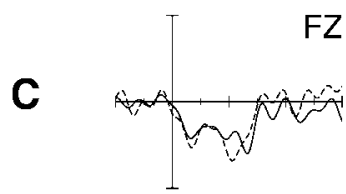

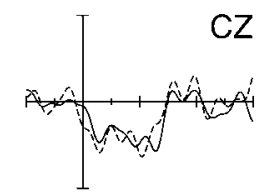

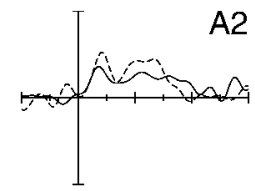

A2

D
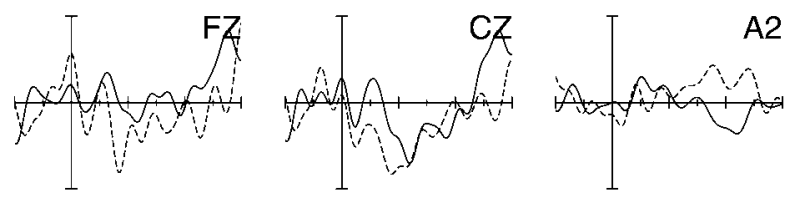

\section{standard}

deviant

Fig. 4. Auditory event-related potentials elicited by music syntactically irregular chords (grand average waveforms from FZ, CZ, and A2), separately for the fifth position (left) and the third position (right) of a chord sequence. As in figure 3, each panel shows auditory event-related brain potentials (AERPs) recorded during the awake state (level A), light sedation (level B), deep sedation (level C), and unconsciousness (level D). Vertical lines indicate the stimulus onset. Short arrows indicate the early right anterior negativity (ERAN) evoked by irregular chords (dotted line) compared with regular chords (solid line). Note the polarity inversion at mastoidal leads (indicated by long arrows). The ERAN was considerably larger at the fifth compared with the third position. As for the mismatch negativity, increasing sedation with propofol resulted in a decrease in ERAN amplitude.

Visual inspection yields that the latencies of P1, MMN, and ERAN increase with increasing sedation (table 3). This observation was not statistically tested because progressing sedation resulted in a decrease of the signal-tonoise ratio; given the number of trials per condition, peak latencies in individual subjects could therefore not reliably be determined.

\section{Discussion}

During the awake state, both stimulus types (tones and chords) elicited distinct AERP effects compared with standard tones: frequency deviants elicited an MMN, and harmonically irregular chords (compared to regular chords) elicited an ERAN. Both MMN and ERAN were elicited during levels of light and deep sedation; the amplitude decreases during increasing propofol sedation did not differ between MMN and ERAN. Both components disappeared during unconsciousness. This finding indicates (1) that the mechanisms underlying physical and music syntactic auditory irregularity detection function even under deep sedation (supporting the hypothesis that the ERAN can be elicited preattentively ${ }^{7,10}$ ) and (2) that these processes are uniformly affected by increasing propofol sedation.

In contrast, the amplitude of the P1 did not differ between wakefulness and sedation, and the P1 was present (but markedly reduced) during unconsciousness, indicating that MMN and ERAN are affected differently by sedation compared with the P1. The behavior of the P1 amplitude (unaffected by sedation but markedly decreased during unconsciousness) indicates that the P1, in contrast with MMN and ERAN, may discriminate drug-induced sedation from adequate anesthesia. Our results concur with those of previous studies investigating dose-dependent effects of increasing anesthesia on midlatency auditory evoked potentials (MLAEPs): marked changes in MLAEPs have been demonstrated for 
propofol concentrations causing unconsciousness, ${ }^{23,24}$ whereas only little is known about the effects of sedative propofol concentrations on MLAEP components. MLAEP recordings during anesthesia with volatile anesthetics showed that sedative drug concentrations did not affect the P1. ${ }^{25,26}$ Moreover, intraoperative AERP recordings with electrodes implanted in the Heschl gyrus demonstrated only weak effects of even deep anesthesia on early cortical responses to auditory stimuli. ${ }^{27}$ Therefore, the observation that the amplitude of the $\mathrm{P} 1$ remains stable during sedation supports the assumption that significant changes in MLAEPs occur only at concentrations causing unconsciousness, whereas late cortical responses are already affected by sedative drug concentrations. ${ }^{28-31}$

In contrast to the virtually unchanged P1, both MMN and ERAN were affected by sedation. We expected the strongest effect of sedation on the ERAN because the ERAN (in contrast with the P1 and the MMN; see introduction, third paragraph) originates mainly from the frontal (inferior frontolateral) cortex. ${ }^{16}$ Contradicting our initial hypothesis, MMN and ERAN are uniformly affected by propofol, supporting the notion of the importance of the frontal cortex in the generation of the MMN: although the MMN mainly originates from the temporal lobe, frontal contributions have been reported. ${ }^{12,14,32,33}$ Moreover, lesions of the (anterior) frontal cortex $^{13,34}$ as well as anterior frontal cortex deactivation during physiologic sleep ${ }^{35}$ cause an attenuation of the MMN.

The frontal cortex plays a crucial role in higher-level cognition (e.g., language perception, memory, attention, behavioral inhibition, [pre]motor functions). That propofol differentially affects the investigated AERP amplitudes shows that cognitive functions that are strongly dependent on neural mechanisms located in the frontal cortex (reflected in ERAN and MMN) are affected even by light propofol sedation, whereas basic cognitive functions located in the primary auditory cortex (reflected in the P1) remain unaffected even under deep sedation. This finding strongly suggests that the cerebral cortex is not globally affected by propofol sedation but that propofol has different effects on different cortical structures. This assumption is corroborated (1) by recent findings showing that low doses of propofol exhibit cognitive effects by preferential decreases of cerebral blood flow in the anterior frontal cortex ${ }^{4}$ and (2) by our finding that a P3a was present only during wakefulness and light sedation. The generation of the $\mathrm{P} 3 \mathrm{a}$ involves more cognitive processes than the MMN (a P3a after an MMN is thought to reflect an involuntary shift of atten$\operatorname{tion}^{8}$ ), and it is assumed that the generation of the $\mathrm{P} 3 \mathrm{a}$ is strongly dependent on neural sources located in the frontal cortex. ${ }^{8}$

Our data indicate a breakdown of cognitive processes that involve the frontal cortex at BIS values between 70 and 50. With respect to the data analysis of the behav- iorally different subgroups during deep sedation (the subgroup with a mean BIS value of 69.6 showed only a marginally present MMN and ERAN; see Results, Eventrelated Potentials: MMN and ERAN, third paragraph), it is well possible that auditory memory processes and processes of music syntactic analysis (as well as language processing; see introduction, fourth paragraph, sixth sentence) collapse at a BIS value just below 70. This idea is supported by studies demonstrating a lack of recall and a loss of responsiveness at BIS values around 70. ${ }^{36-39}$ However, further investigations are necessary to reveal the exact BIS value at which MMN and ERAN (respective auditory sensory memory operations and musical processing) collapse.

Sedation in spontaneously breathing patients is usually accompanied by an increase in arterial carbon dioxide concentration, which could have affected our results. Increased arterial carbon dioxide tension $\left(\mathrm{PaCO}_{2}\right)$ values are associated with an altered electrical activity of the brain. Moreover, they are assumed to adversely affect the cognitive performance. However, the increase in $\mathrm{PaCO}_{2}$ caused by propofol in our study should be small. Sedative propofol concentrations have been reported to cause increases in $\mathrm{PaCO}_{2}$ of approximately $5 \mathrm{mmHg}$ during spontaneous respiration. ${ }^{3}$ Such modest increases in $\mathrm{PaCO}_{2}$ affect neither latency nor amplitude of cortical AERPs. ${ }^{40}$

Propofol-induced unconsciousness abolished the MMN and the ERAN and caused a clear reduction of the P1 amplitude, indicating that processes in and in the vicinity of the primary auditory cortex were markedly affected at BIS values around 50. This finding is of clinical relevance because it gives electrophysiologic evidence for the assumption that BIS values between 40 and 60 are associated with loss of auditory perception. ${ }^{36,37}$

In conclusion, the behavior of the investigated AERPs indicates sequential, concentration-dependent effects of propofol on auditory functions. Sedative concentrations first affect auditory change detection processes that involve frontal cortices, whereas processes merely involving the primary auditory cortex are only affected by propofol concentrations causing unconsciousness. Physical and music syntactic auditory irregularity detection processes are similarly affected by increasing propofol doses but can be observed even under deep sedation. The alterations in the recorded AERPs were accompanied by specific changes in the behavioral state. Auditory sensory memory operations and music syntactic processing (and, therefore, presumably also language processing) collapse at the same time, at which loss of responsiveness was observed. At BIS values reflecting adequate anesthesia, only residual signs of auditory perception were found in the primary auditory cortex. The findings strongly suggest that the cerebral cortex is not globally affected by propofol sedation but that propofol has dif- 
ferent effects on cognitive processes mediated by different cortical structures.

\section{References}

1. Fiset P, Paus T, Daloze T, Plourde G, Meuret P, Bonhomme V, Hajj-Ali N, Backman SB, Evans AC: Brain mechanisms of propofol-induced loss of consciousness in humans: A positron emission tomographic study. J Neurosci 1999; 19:5506-13

2. Heinke W, Schwarzbauer C: Subanesthetic isoflurane affects task-induced brain activation in a highly specific manner. ANESTHESIOLOGY 2001; 94:973-81

3. Bonhomme V, Fiset P, Meuret P, Backman S, Plourde G, Paus T, Bushnell MC, Evans AC: Propofol anesthesia and cerebral blood flow changes elicited by vibrotactile stimulation: A positron emission tomography study. J Neurophysiol 2001; 85:1299-308

4. Veselis RA, Reinsel RA, Feshchenko VA, Dnistrian AM: A neuroanatomical construct for the amnesic effects of propofol. ANESTHESIOLOGY 2002; 97:329-37

5. Näätänen R: Attention and Brain Function. Hillsdale, New Jersey, Erlbaum, 1992

6. Schroger E: Measurements and interpretation of the mismatch negativity (MMN). Behav Res Methods Instruments Comput 1998; 30:131-45

7. Koelsch S, Schroger E, Gunter TC: Music matters: Preattentive musicality of the human brain. Psychophysiology 2002; 39:38-48

8. Näätänen R, Alho K, Schröger E: Electrophysiology of attention, Stevens Handbook of Experimental Psychology, 3rd edition. Edited by Pashler H, Wixted J. New York, John Wiley, 2002, pp 601-53

9. Erwin R, Buchwald JS: Midlatency auditory evoked responses: Differential effects of sleep in the human. Electroencephalogr Clin Neurophysiol 1986; 65:383-92

10. Koelsch S, Gunter TC, Schröger E, Tervaniemi M, Sammler D, Friederici AD: Differentiating ERAN and MMN: An ERP study. Neuroreport 2001; 12:1385-9

11. Liégeois-Chauvel C, Musolino A, Badier JM, Marquis P, Chauvel P: Evoked potentials recorded from the auditory cortex in man: Evaluation and topography of the middle latency components. Electroencephalogr Clin Neurophysiol 1994; 92:204-14

12. Giard MH, Perrin F, Pernier J, Bouchet P: Brain generators implicated in the processing of auditory stimulus deviance: A topographic event-related potential study. Psychophysiology 1990; 27:627-40

13. Alho K, Woods DL, Algazi A, Knight RT, Näätänen R: Lesions of frontal cortex diminish the auditory mismatch negativity. Electroencephalogr Clin Neurophysiol 1994; 91:353-62

14. Alho K, Tervaniemi M, Huotilainen M, Lavikainen J, Tiitinen H, Ilmoniemi RJ, Knuutila J, Näätänen R: Processing of complex sounds in the human auditory cortex as revealed by magnetic brain responses. Psychophysiology 1996; 33: 369-75

15. Alho K, Winkler I, Escera C, Huotilainen M, Virtanen J, Jääskeläinen IP, Pekkonen E, Ilmoniemi RJ: Processing of novel sounds and frequency changes in the human auditory cortex: Magnetoencephalographic recordings. Psychophysiology 1998; 35:211-24

16. Maess B, Koelsch S, Gunter TC, Friederici AD: Musical syntax is processed in Broca's area: An MEG study. Nat Neurosci 2001; 4:540-5

17. Schwender D, Kunze-Kronawitter H, Dietrich P, Klasing S, Forst H, Madler C: Conscious awareness during general anaesthesia: Patients perceptions, emotions, cognition and reactions. Br J Anaesth 1998; 80:133-9

18. Patel AD, Gibson E, Ratner J, Besson M, Holcomb PJ: Processing syntactic relations in language and music: An event-related potential study. J Cogn Neurosci $1998 ; 10: 717-33$

19. Janata $\mathrm{P}$, Tillmann $\mathrm{B}$, Bharucha JJ: Listening to polyphonic music recruits domain-general attention and working memory circuits. Cogn Affect Behav Neurosci 2002; 2:121-40
20. Hahne A, Friederici AD: Electrophysiological evidence for two steps in syntactic analysis: Early automatic and late controlled processes. J Cogn Neurosci 1999; 11:194-205

21. Billard V, Gambus PL, Chamoun N, Stanski DR, Shafer SL: A comparison of spectral edge, delta power, and Bispectral Index as EEG measures of alfentanil, propofol, and midazolam drug effect. Clin Pharmacol Ther 1997; 61:45-58

22. Koelsch S, Gunter TC, Friederici AD, Schröger E: Brain indices of music processing: 'Non-musicians' are musical. J Cogn Neurosci 2000; 12:520-41

23. Chassard D, Joubaud A, Colson A, Guiraud M, Dubreuil C, Banssillon V: Auditory evoked potentials during propofol anaesthesia in man. $\mathrm{Br} \mathrm{J}$ Anaesth 1989; 62:522-6

24. Thornton C, Konieczko KM, Knight AB, Kaul B, Jones JG, Doré CJ, White DC: Effect of propofol on the auditory evoked response and oesophageal contractility. Br J Anaesth 1989; 63:411-7

25. Schwender D, Conzen P, Klasing S, Finsterer U, Pöppel E, Peter K: The effects of anesthesia with increasing end-expiratory concentrations of sevoflurane on midlatency auditory evoked potentials. Anesth Analg 1995; 81:817-22

26. Schwender D, Klasing S, Conzen P, Finsterer U, Pöppel E, Peter K: Midlatency auditory evoked potentials during anaesthesia with increasing endexpiratory concentrations of desflurane. Acta Anaesthesiol Scand 1996; 40:171-6

27. Howard MA, Volkov IO, Mirsky R, Garrell PC, Noh MD, Granner M, Damasio H, Steinschneider M, Reale RA, Hind JE, Brugge JF: Auditory cortex on the posterior superior temporal gyrus. J Comp Neurol 2000; 416:79-92

28. Simpson TP, Manara AR, Kane NM, Barton RL, Rowlands CA, Butler SR: Effect of propofol anaesthesia on the event-related potential mismatch negativity and the auditory-evoked potential N1. Br J Anaesth 2002; 89:382-8

29. Yppärilä H, Karhu J, Westerén-Punnonen S, Musialowicz T, Partanen J: Evidence of auditory processing during postoperative propofol sedation. Clin Neurophysiol 2002; 113:1357-64

30. Reinsel RA, Veselis RA, Wronski M, Marino P: The P300 event-related potential during propofol sedation: A possible marker for amnesia? Br J Anaesth $1995 ; 74: 674-80$

31. Veselis RA, Reinsel RA, Feshchenko VA: Drug-induced amnesia is a separate phenomenon from sedation: Electrophysiologic evidence. Anesthesiology 2001; 95:896-907

32. Alain C, Achim A, Woods DL: Separate memory-related processing for auditory frequency and pattern. Psychophysiology 1999; 36:737-44

33. Opitz B, Rinne T, Mecklinger A, von Cramon DY, Schröger E: Differential contributions of frontal and temporal cortices to auditory change detection: fMRI and ERP results. Neuroimage 2002; 15:167-74

34. Alain C, Woods DL, Knight RT: A distributed cortical network for auditory sensory memory in humans. Brain Res 1998; 23:23-37

35. Atienza M, Cantero JL, Dominguez-Marin E: Mismatch negativity (MMN): An objective measure of sensory memory and long-lasting memories during sleep. Int J Psychophysiol 2002; 46:215-25

36. Glass PS, Bloom M, Kearse L, Rosow C, Sebel P, Manberg P: Bispectral analysis measures sedation and memory effects of propofol, midazolam, isoflurane, and alfentanil in healthy volunteers. ANESTHESIOLOGY 1997; 86:836- 47

37. Kerssens C, Klein J, van der Woerd A, Bonke B: Auditory information processing during adequate propofol anesthesia monitored by electroencephalogram Bispectral Index. Anesth Analg 2001; 92:1210-4

38. Flaishon R, Windsor A, Sigl J, Sebel PS: Recovery of consciousness after thiopental or propofol: Bispectral Index and isolated forearm technique. ANEsTHESIOLOGY 1997; 86:613-9

39. Kearse LA Jr, Rosow C, Zaslavsky A, Connors P, Dershwitz M, Denman W: Bispectral analysis of the electroencephalogram predicts conscious processing of information during propofol sedation and hypnosis. ANesthesiology 1998; 88: 25-34

40. Bloch-Salisbury E, Lansing R, Shea SA: Acute changes in carbon dioxide levels alter the electroencephalogram without affecting cognitive function. Psychophysiology 2000; 37:418-26 